[0212-7199 (2007) 24: 2; pp 55-56 ANALES DE MEDICINA INTERNA Copyright $@ 2007$ ARAN EDICIONES, S.L.

AN. MED. INTERNA (Madrid) Vol. 24, N. $^{\circ} 2$, pp. $55-56,2007$

\title{
Los ultrasonidos, la densitometría y la osteoporosis
}

Gómez de Tejada Romero MJ, Sosa Henríquez. M. Los ultrasonidos, la densitometría y la osteoporosis. An Med Interna (Madrid) 2007; 24: $55-56$.

La osteoporosis es una enfermedad extraordinariamente frecuente, que afecta tanto a hombres como a mujeres, y que produce fracturas como principal complicación clínica (1), siendo la fractura vertebral la más frecuente y la de cadera o extremidad proximal del fémur la más grave, por su elevada morbilidad y no despreciable mortalidad (2). La prevención y el tratamiento de la osteoporosis generan un considerable consumo de recursos sanitarios de todo tipo (3).

Hoy en día sabemos que la resistencia al hueso frente a las fracturas viene condicionada, por una parte, por la cantidad de masa ósea, y por otra, por la calidad del hueso (4). Y si bien la cantidad puede medirse actualmente de una manera muy fiable con varias técnicas no invasivas, no ocurre lo mismo con la calidad ósea. En principio, bajo este concepto se agrupan componentes muy diferentes del hueso: el remodelado óseo, la estructura geométrica, la conectividad de las trabéculas, etc. (5), difícil de evaluar con una sola técnica; por lo que, en la actualidad, la evaluación de la resistencia ósea se realiza estimando sólo la cantidad, es decir, extrapolando los datos densitométricos y considerando los mismos como una valoración representativa de aquélla, a falta de otras técnicas más apropiadas.

La absorciometría radiológica dual (DXA), conocida genéricamente como densitometría ósea, está considerada como el "patrón oro" en la medición de la densidad mineral ósea (DMO), y es la técnica de elección para establecer el diagnóstico de osteoporosis, basado en los criterios de la OMS (6). Sin embargo, esta asimilación de equivalencia entre resistencia ósea y DMO da lugar a algunos problemas en la práctica clínica diaria. Así, por ejemplo, sabemos que un buen número de pacientes que tienen osteoporosis densitométrica no llegan a fracturarse nunca y por el contrario, una proporción no despreciable de pacientes tienen fracturas con valores densitométricos de osteopenia e incluso normales $(7,8)$.

Efectivamente, la DXA es un método cuantitativo de gran exactitud $(1-3 \%)$ y precisión $(<1 \%)(9)$, con una gran rapidez de exploración (segundos) y de muy baja radiación $(<5 \mathrm{mRem})$, que permite la medición de la DMO en aquellas zonas donde es más relevante la fractura osteoporótica (columna lumbar y fémur proximal), además de cuantificar objetivamente el riesgo de fractura $(10,11)$. Pero entre sus inconvenientes está el alto coste económico, la necesidad de una ubicación amplia y con vigilancia radiológica y un personal técnico adiestrado para su manejo, y lo que es más importante, la incapacidad para medir los aspectos cualitativos del hueso que participan en la etiopatogenia de la fractura osteoporótica. Por eso, otras técnicas han ido surgiendo paralelamente para soslayar estos inconvenientes: entre las técnicas alternativas destacan la ultrasonografía cuantitativa (QUS), que ha evolucionado de tal manera que hoy en día se ha hecho, por méritos propios, un lugar en el campo del diagnóstico de la osteoporosis (12).

Respecto a los QUS, la ventaja principal es que se trata de una técnica absolutamente inocua (13), cuyos aparatos son pequeños y portátiles, muy cómoda y sencilla de realizar, que no precisa de una preparación ni titulación específica de sus operarios, de bajo coste económico, y también muy rápida.

Pero los QUS tienen también sus inconvenientes. Uno de ellos es la baja precisión o elevado coeficiente de variación, que le impide ser utilizada para seguimientos y estudios longitudinales (14). Además, aunque existe una cierta correlación entre ambas técnicas (15), no podemos aplicar los criterios de la OMS, definidos específicamente para la DXA, a la QUS, debido a que son medidas de distintos aspectos del hueso, el cual se comporta de distinta forma ante medios físicos distintos (rayos X y ultrasonidos) $(16,17)$. No obstante, el hecho de que no sea equiparable a la DXA no invalida a la QUS como método de valoración en la osteoporosis. Numerosos estudios han demostrado que la QUS tiene capacidad para predecir el riesgo de fracturas osteoporóticas (18-20), y puesto que los parámetros QUS no miden cantidad ósea (DMO) como hace la DXA, es de suponer que, si predicen el riesgo de fractura, estén valorando otros aspectos determinantes de la resistencia ósea que estén relacionados con la calidad del hueso (21).

Por ello, se han efectuado algunos estudios tratando de averiguar si la combinación de estas dos técnicas mejoran la sensibilidad de ambas por separado, ya que una mide cantidad (DXA) y la otra calidad (QUS). Los resultados han sido contra- 
dictorios $(22,23)$, aunque parece ser que la tendencia generalizada es a considerar que no se consigue una mejor resultado en la predicción del riesgo de fractura al combinar ambas técnicas.

De todas formas, la QUS es una técnica de valoración ósea que tiene muy buenas cualidades para generalizar su empleo en la práctica clínica (24), pero cuya verdadera traducción está por definir. Quizás fuera necesario hacer estudios dirigidos para comprender mejor qué aspectos mide realmente del hueso, sin querer aplicarle los esquemas aprendidos de la DXA.

\section{Bibliografía}

1. NIH Consensus Panel: Consensus Development Conference: Diagnosis, Prophylaxis and Treatment of Osteoporosis. Am J Med 1993; 94: 646-50.

2. Cummings SR, Melton LJ III. Epidemiology and outcomes of osteoporotic fractures. Lancet 2002; 359: 1761-7.

3. Johnell O, Kanis JA. An estimate of the worldwide prevalence and disability associated with osteoporotic fractures. Osteoporos Int 2006; 17 : 1726-33

4. NIH Consensus Development Panel on Osteoporosis Prevention, Diagnosis and Therapy. JAMA 2001; 285: 785-95.

5. Recker RR, Barger-Lux MJ. The elusive concept of bone quality. Curr Osteoporos Rep 2004; 2: 97-100.

6. World Health Organisation. Assessment of fractures risk in screening for osteoporosis. WHO technical report series 843. Geneva: WHO, 1994.

7. Sosa Henríquez M, Hernández Hernández D, Castro Medina R, Travesí García I, Sablón González N, Sarmiento Santana J. Las mujeres postmenopáusicas con osteopenia densitométrica tienen una elevada prevalencia de fracturas. Rev Esp Enf Metab Óseas 2005; 14: 110.

8. M. Sosa Henríquez, MJ Gómez de Tejada Romero. El término osteopenia y el riesgo de fractura. An Med Interna (Madrid) 2006; 23: 1-3.

9. Gómez Alonso C, Bernardino Díaz J, Cannata Andía J. Metodología de la evaluación de la masa ósea. En: Díaz Curiel M, Díez Pérez M, Gómez Alonso C, eds. Nuevas fronteras en el estudio de la densidad ósea en la población española. Madrid: FHOEMO, SEIOMM, RPR 1996; $11-55$

10. Kanis JA, Johnell O, Oden A, Johansson H, Eisman, JA, Fujiwara S, et al. The use of multiple sites for the diagnosis of osteoporosis. Osteoporos Int 2006; 17: 527-34.

11. Johnell O, Kanis JA, Oden A, Johansson H, de Laet C, Delmas P, et al. Predictive value of BMD for hip and other fractures. J Bone Miner Res 2005; 20: 1185-94.

12. Gluer $\mathrm{CC}$ for the International Quantitative Ultrasound Consensus Group. Quantitative ultrasound techniques for the assessment of osteoporosis: expert agreement on current status. J Bone Miner Res 1997; 12: 1280-8.

13. Bioeffects considerations for the safety of diagnostic ultrasound. American Institute of Ultrasound in Medicine. Bioeffects Committee. J Ultrasound Med 1988; 7 (Supl. 9): S1-38.

\section{J. GÓMEZ DE TEJADA ROMERO, M. SOSA HENRÍQUEZ}

Departamento de Medicina. Universidad de Sevilla. ${ }^{1}$ Universidad de Las Palmas de Gran Canaria. Grupo de Investigación en Osteoporosis. Hospital Universitario Insular. Servicio de Medicina Interna. Unidad Metabólica Ósea. Las Palmas de Gran Canaria

14. Sosa M, Saavedra P, Muñoz-Torres M, Alegre J, Gómez-Alonso C González-Macías J, et al. for the GIUMO Study Group. Quantitative Ultrasound Calcaneus Measurements: Normative Data and Precision in Spanish Population. Osteoporos Int 2002; 13: 487-92.

15. He YQ, Fan B, Hans D, Li J, Wu CY, Njeh CF, et al. Assessment of a new quantitative ultrasound calcaneus measurement: precision and discrimination of hip fractures in elderly women compared with Dual XRay Absorptiometry. Osteoporos Int 2000; 11: 354-69.

16. Frost ML, Blake GM, Fogelman I. Can the WHO criteria for diagnosing osteoporosis be applied to calcaneal quantitative ultrasound? Osteoporos Int 2000; 11: 321-30.

17. Gómez de Tejada Romero MJ, Sosa Henríquez M. Los ultrasonidos, la densitometría, el T-score y los criterios de la OMS para el diagnóstico de la osteoporosis. Rev Esp Enf Metab Óseas 2002; 11: 165-6.

18. Hernández JL, Marín F, González-Macías J, Díez-Pérez A, Vila J, Giménez S, et al. Discriminative capacity of calcaneal quantitative ultrasound and of osteoporosis and fracture risk factors in postmenopausal women with osteoporotic fractures. Calcif Tissue Int 2004; 74: 357-65.

19. Marín F, González-Macías J, Díez-Pérez A, Palma S, Delgado-Rodríguez M. Relationship between bone quantitative ultrasound and fractures: a meta-analysis. J Bone Miner Res 2006; 21: 1126-35.

20. Sosa M, Saavedra P, Del Pino-Montes J, Alegre J, Pérez-Cano R, Martínez Díaz Guerra G, et al. Postmenopausal women with Colles' fracture have lower values of bone mineral density than controls as measured with Quantitative Ultrasound and Densitometry. J Clin Densitom 2005; 8: 430-5.

21. Njeh CF, Fuerst T, Diessel E, Genant HK. Is quantitative ultrasound dependent on bone structure? A reflection. Osteoporos Int 2001; 12: 1-15.

22. Gonnelli S, Cepollaro C, Gennari L, Montagnani A, Caffarelli C, Merlotti $\mathrm{D}$, et al. Quantitative ultrasound and dual-energy X-ray absorptiometry in the prediction of fragility fracture in men. Osteoporos Int 2005; 16: 963-8.

23. Peretz A, De Maertelaer V, Moris M, Wouters M, Bergmann P. Evaluation of quantitative ultrasound and dual X-ray absorptiometry measurements in women with and without fractures. J Clin Densitom 1999; 2: 127-33.

24. Díez-Pérez A, Marín F, Vila J, Abizanda M, Cervera A, Carbonell C, et al. Evaluation of calcaneal quantitative ultrasound in a primary care setting as a screening tool for osteoporosis in postmenopausal women. J Clin Densitom 2003; 6: 237-45. 